\title{
Factores psicosociales y culturales en la prevención y tratamiento de las enfermedades de transmisión sexual
}

\author{
Psychosocial and cultural factors in the prevention \\ and treatment of sexually transmitted diseases
}

\footnotetext{
1 Centro de Estudios de Estado y Sociedad, Consejo Nacional de Investigaciones Científicas y Técnicas. Sánchez de Bustamante 27 1173 Buenos Aires, Argentina. monik@cedes.edu.ar
}

\begin{abstract}
The article deals with the psychosocial and cultural dimensions of sexually transmitted diseases. Based on results from a qualitative study with lower-class males and females (young and adult) from a neighbourhood in the metropolitan area of Buenos Aires, the author discusses how lay beliefs (about symptoms, transmission, consequences) and norms regarding sexual matters and gender relations affect people's ability to consider themselves at risk and/or adopt preventive behaviors. Implications of research results for the design of culturally appropriate strategies to promote sexual and reproductive health are also provided.
\end{abstract}

Key words Sexually Transmitted Diseases; Gender; Sex Behavior

Resumen El artículo trata de las dimensiones psicosociales y culturales de las enfermedades de transmisión sexual. Sobre la base de resultados de una investigación cualitativa realizada entre hombres y mujeres de sectores populares (jóvenes y adultos) en el Gran Buenos Aires, la autora discute cómo las nociones legas acerca de las ETS (sus síntomas, vías de transmisión, consecuencias) y las normas culturales sobre el comportamiento sexual y las relaciones de género afectan la habilidad de las personas para considerarse en riesgo y/o adoptar conductas de prevención. También se discuten las implicancias de estos hallazgos en términos de estrategias apropiadas para promover conductas de cuidado de la salud sexual y reproductiva.

Palabras clave Enfermedades Sexualmente Transmisibles; Género; Comportamiento Sexual 


\section{Introducción}

Estas notas presentan resultados de una investigación realizada en colaboración con Silvina Ramos y Edith Pantelides y financiada por la Organización Mundial de la Salud. Esa investigación, realizada en Buenos Aires en 1994, tenía por objetivo producir conocimiento acerca de los factores psicosociales y culturales que inciden en la prevención y tratamiento de las enfermedades de transmisión sexual (en adelante ETS). Dado que la problemática había sido escasamente tratada en nuestro medio, nos interesaba relevar los conocimientos, creencias, opiniones y preocupaciones de varones y mujeres, jóvenes y adultos acerca de estas enfermedades. Cuáles eran las ETS más conocidas, cuáles las ideas predominantes acerca de las formas de transmisión, la prevención y el tratamiento, si existía percepción de riesgo o vulnerabilidad y si ésta era diferente según sexo/género eran algunos de los interrogantes con que iniciamos nuestro trabajo. Nuestro propósito era comprender los obstáculos a la prevención y el tratamiento de estas enfermedades, produciendo así información de utilidad para el diseño de actividades educativas culturalmente apropiadas.

\section{Marco teórico-conceptual}

Recogiendo la experiencia de las investigaciones acerca de los factores que favorecen o dificultan la prevención del VIH/SIDA, se utilizó un marco conceptual heterodoxo, que recuperó e integró conceptos provenientes de diferentes disciplinas y perspectivas teóricas (i.e. la antropología médica, la educación para la salud, las teorías constructivistas acerca de la sexualidad, el feminismo). Partimos de la hipótesis de que la prevención y el tratamiento de las ETS son afectados por dos conjuntos de factores, estrechamente interrelacionados, que de manera global denominamos "salud/enfermedad" y "género/sexualidad". El primero engloba las nociones legas o populares acerca de las ETS y el VIH/SIDA: creencias acerca de las formas de transmisión, la posibilidad de prevenirlas, las consecuencias atribuidas, entre otras, que son productos de desarrollos culturales nativos o populares, y que no derivan explícitamente del arco conceptual de la medicina moderna (Foster \& Anderson, 1978). Estas nociones - pragmáticas, de origen sincrético, flexibles - son la base argumentativa que las personas utilizan para interpretar sus percepciones y sensaciones y orientar y evaluar las accio- nes que emprenden. Dado que las ETS involucran la sexualidad, suponíamos que esas nociones estarían fuertemente atravesadas por normas y prescripciones culturales acerca de las identidades y relaciones de género y el ejercicio de la sexualidad. Nuestro marco conceptual recogía así el conocimiento acumulado en la temática, el cual indica que las interacciones sexuales y la propia forma de entender la sexualidad están intrínsecamente ligadas a los conceptos culturales de la femineidad y la masculinidad (Dixon-Mueller, 1993).

\section{Notas metodológicas}

Dados los objetivos de la investigación y su carácter exploratorio y descriptivo, optamos por una aproximación cualitativa, la que es especialmente adecuada cuando el investigador tiene ideas preliminares sobre el tema de estudio pues permite descubrir lo que es importante para la población y por qué (Zeller, 1990). Se utilizaron dos técnicas de recolección de información: el grupo focal - ya utilizado en la literatura para explorar los conocimientos de la población respecto de las ETS (entre otros, Helitzer-Allen y Allen, 1994) - y la entrevista semiestructurada. Se utilizó la combinación de técnicas a fin de aumentar la confiabilidad de la información ya que se trataba de una cuestión no sólo poco explorada sino también muy sensitiva (Helitzer-Allen et al., 1994). Con ambas técnicas, entonces, se cubrieron los mismos temas o preguntas.

En la entrevista, además, se aplicaron dos técnicas sistemáticas provenientes de la antropología cognitiva destinadas a aislar o definir dominios culturales o semánticos - es decir, conjuntos organizados de palabras, conceptos o frases que refieren a una esfera conceptual determinada: el listado de libre enumeración (free list) y el agrupamiento en pilas (pile sort). En los grupos y entrevistas se solicitó a los participantes que listaran los problemas de salud, molestias o enfermedades "relacionados con la vida sexual”. Luego, para cada una de las enfermedades o problemas mencionados, se indagó acerca de síntomas, formas de prevención, posibilidades de cura, tratamientos, etc. También se preguntó por las fuentes a través de las cuales se había obtenido dicha información, las personas con quienes habitualmente se habla de estos temas y las consecuencias que tienen las ETS para varones y mujeres y para las parejas. Hacia el final de la entrevista se entregaba un conjunto de tarjetas que contenían los nombres de ETS obtenidos en los diversos grupos 
focales, las que eran leídas por el(la) entrevistador(a). Se solicitaba entonces a cada persona que separara las tarjetas en dos pilas según hubiera oído o no hablar de cada una de ellas. Luego se le pedía que agrupara las que conocía con la consigna de "poner juntas las que van juntas (tienen algo en común)".

El trabajo de campo se realizó en un barrio popular de un partido del Gran Buenos Aires en el que viven unas 600 familias. Se realizaron 10 grupos focales ( 5 de mujeres y 5 de varones) y 20 entrevistas semi-estructuradas (10 con mujeres y 10 con varones) con jóvenes (18 a 25) y adultos (35 a 50). La población entrevistada tenía básicamente educación primaria (completa e incompleta) y se desempeñaba en ocupaciones de servicios de baja calificación.

Los grupos estaban constituidos por entre 8 y 10 personas, reclutadas por una líder comunitaria a quien el equipo conocía de una experiencia de trabajo anterior en el barrio. Los grupos focales fueron conducidos por un/a moderador/a y un/a secretario/a del mismo sexo que los participantes. Las sesiones (que fueron grabadas y luego transcritas y codificadas) tuvieron una duración de algo más de una hora y se desarrollaron en el centro de salud del barrio en horarios de escasa concurrencia. Tanto en los grupos como en las entrevistas se remuneró a los participantes con una pequeña suma de dinero como compensación por el tiempo dedicado.

Al inicio de la investigación se entrevistó a tres médicos que trabajan en la zona (un infectólogo y dos ginecólogos, uno de ellos especialista en adolescencia) con la intención de tener una aproximación a la perspectiva de los profesionales sobre la temática en estudio.

\section{Resultados}

El principal hallazgo del estudio es que la manera en que estas enfermedades aparecen en el imaginario popular dista considerablemente de la perspectiva biomédica. Veamos brevemente algunos de los rasgos dominantes de la caracterización popular acerca de la ETS.

Los listados ofrecidos espontáneamente por varones y mujeres incluyeron ETS, síntomas, fluidos corporales, problemas ginecológicos e incluso enfermedades que no son de transmisión sexual. Fueron marcadas, además, las diferencias entre las respuestas según sexo. Con excepción del SIDA, mencionado por todos los participantes del estudio, varones y mujeres espontáneamente mencionaron diferentes ETS: “la pudrición” y la ladilla, ellos; la sífilis y las infecciones (en general, vaginales, urinarias), ellas. El dato no debe ser interpretado linealmente como "diferencias en el conocimiento”. El pile-sort reveló que la mayoría de las mujeres conocía el término "pudrición", aunque no lo habían mencionado espontáneamente. Se pudo observar que, en términos generales, los varones tenían más información que las mujeres acerca de la posibilidad de prevenir las ETS y de la forma de tratarlas.

Las ETS fueron reiteradamente asociadas a la falta de higiene (personal, de sábanas y toallas, de los baños) y al contacto sexual con personas que "no se conocen". Fue llamativa la insistencia con que ambos géneros $y$, en particular los varones se refirieron a las ETS como enfermedades transmitidas por las mujeres. Algunos de los entrevistados relacionaron estas enfermedades con una categoría particular de mujeres ("de la calle", "de la noche", "sucias") pero la mayoría de ellos mencionaron que amigos y conocidos se habían contagiado de una ETS de una mujer (a secas). Las expresiones populares transmiten la idea de que las mujeres hacen daño, "pudren" o "pasan" las ETS a los varones.

"La menstruación fuerte que tienen las mujeres es lo que te produce todas esas enfermedades" (varón, 42 años).

"Tengo conocidos que han estado enfermos de hacer uso sexual con una mujer" (varón, 46 años).

“La pudrición es cuando estás haciendo el amor. La mina tiene un flujo fuerte y vos estás débil y te pudrís, por el flujo fuerte que ella tie$n e "$ (varón, 20 años).

"Decían que agarrás una piba y después te salen hongos" (varón, grupo de jóvenes).

En general, las ETS no son percibidas como enfermedades que pueden tener consecuencias sobre la salud o la capacidad reproductiva. El conflicto de pareja, los celos y la desconfianza fueron la principal consecuencia identificada por ambos géneros, si bien los varones mencionaron también la disminución del deseo sexual y/o la interferencia con la dinámica sexual.

El estudio evidenció claramente que aún existiendo percepción de riesgo (que los varones anclan en la "peligrosidad de los fluidos femeninos" y las mujeres en la "natural infidelidad" de sus parejas) no necesariamente se adoptan conductas adecuadas para prevenir las ETS. La escasa disponibilidad y capacidad para el "sexo más seguro" remite a una compleja trama compuesta de normas y valores, estereotipos, relaciones de poder, sentimientos (afecto, temor y vergüenza) y significados (i.e. 
que el preservativo "no es para el matrimonio”). Así, por ejemplo, resultó evidente que los mandatos culturales que definen la sexualidad masculina como "indomable" (el varón no debe desaprovechar oportunidades de contacto sexual) y/o postulan que correr riesgos es un elemento esencial de la masculinidad conspiran seriamente contra la capacidad de los varones de protegerse y proteger a sus parejas sexuales.

"El hombre se manda igual. 'Yo soy hombre - dice - y lo hago'” (varón, grupo de adultos).

"Es un momento que le tenés que dar, si la mina se te entrega. En el momento no se va a pensar nada, creo yo. Te mandás igual, porque otra no te queda" (varón, grupo de jóvenes).

A su vez, los estereotipos y relaciones de género proveen a las "señoras de su casa" una protección imaginaria o afectan seriamente la capacidad de aquellas que se perciben en riesgo de decidir acerca de cómo procederán los encuentros sexuales. Muchas de las mujeres del estudio no creían que fuera necesario usar preservativo con sus parejas estables y otras declararon que no se animarían a proponerlo. Los temores acerca de la eventual reacción masculina y la vergüenza fueron las principales razones señaladas.

“¿Para qué usarlo si es el marido?” (mujer, grupo de jóvenes).

"No me animaría a decirle que use preservativo. Yo hace 11 años que estoy con mi marido... y lo conozco" (mujer, grupo de jóvenes).

P6: Es lo mismo que a tu marido le vayas y le digas que tenés que usar un preservativo...

PX: Claro.

P6: Yo no me animaría, no sé, pienso que me saca rajando...

Mo: Que te imaginás que pasaría?

P6: ...pienso que él se negaría, por él, no por mi, porque yo voy y soy directa y le digo, pero y él...claro, claro (risas).

P5: Aparte va a pensar que una no le tiene confianza...

PX: Claro.

P10: Si no le tenés confianza a tu marido, no sé...(mujeres, grupo de jóvenes).

Finalmente, para completar este breve presentación de los principales resultados de la investigación, una breve mención a la distinción ETS/VIH-SIDA. Los/as entrevistados/as tenían mayor información e información de mejor calidad acerca del VIH/SIDA que de las ETS. No obstante ello, se detectaron confusiones y creencias erróneas. Entre otras, que sólo los enfermos contagian; que los enfermos de SIDA no contagian porque están muy débiles; que sólo la mujer o el varón contagian; que el
VIH se transmite por compartir un cigarrillo de marihuana o aspirar drogas; que la sangre infectada puede "entrar por los poros".

\section{Discusión}

Nuestros resultados son coincidentes con los de numerosos estudios socio-antropológicos acerca de las ETS/el VIH-SIDA. Las similitudes incluyen tanto la manera en que la población concibe a las ETS (Desormeáux et al., 1992) como lo que podríamos llamar las estrategias "legas” de prevención. En particular, la idea de que "conocer a la persona" con la que se tienen relaciones sexuales es una forma eficaz de protección (entre otros, Paiva, 1993). Nuestros hallazgos también abonan la idea de que los estereotipos de género y las relaciones de poder entre varones y mujeres afectan centralmente la capacidad de las personas de adoptar conductas adecuadas para prevenir las ETS (Gupta \&Weiss, 1993; Carovano, 1995; Campbell, 1995). ¿Qué implicancias para la prevención es posible derivar de estos resultados?

En primer lugar, que los mensajes educativos son siempre interpretados y resignificados en función de un complejo modelo en el que coexisten creencias más o menos compatibles con la perspectiva biomédica y que, por lo tanto, las estrategias de prevención deben ser sensibles a la lógica y a los significados subyacentes en las nociones legas acerca de estas enfermedades (i.e. representaciones acerca de los fluidos corporales, la polución, lo frío y lo caliente).

En segundo lugar, que la transmisión de información debe ir acompañada de una discusión abierta acerca de las normas sexuales y los estereotipos de género que estimule y legitime la emergencia y el procesamiento de las emociones y los prejuicios. Aumentar la capacidad de comunicación en torno a cuestiones vinculadas a la sexualidad es clave. Por ser la sexualidad una faceta del comportamiento humano atravesada por prohibiciones, tabúes y prejuicios, proveer a varones y mujeres espacios "separados” para conversar estas cuestiones puede ser la estrategia más apropiada para promover dicha comunicación entre los miembros de la pareja. Acordamos con Campbell (1995) en que los hombres deben ser enfocados directamente como parejas sexuales masculinas (i.e. en los lugares de trabajo, en la comunidad), en vez de aproximarse a ellos a través de las mujeres. Centrar los esfuerzos exclusivamente en el mejoramiento de las habilidades de las mujeres para negociar, particularmente en el caso 
de las mujeres casadas, resulta no sólo ineficaz sino fundamentalmente inequitativo. En base a nuestra experiencia, sugerimos que la promoción del "sexo más seguro" se ancle en las ventajas del preservativo como anticonceptivo dado que tanto varones como mujeres suelen expresar opiniones negativas respecto de la píldora y sus eventuales efectos secundarios y que algunos varones otorgan mayor legitimidad al preservativo como medio de evitar embarazos no deseados que de "evitar un contagio”. Sensibilizar a los varones acerca de la importancia del uso del condón en relaciones secundarias podría ser una estrategia a utilizar, ya que esta parece ser un práctica bastante difundida. Finalmente, respecto del trabajo con las mujeres, sugerimos intervenciones que incluyan en la agenda el conjunto de cuestiones relevantes para su salud reproductiva, que recuperen la sexualidad y promuevan la conciencia crítica acerca de su condición de género. Aumentar la accesibilidad del condón femenino (i.e. a través de los centros de salud) podría ser una estrategia a explorar, dada la generalizada aceptación que recibió entre las mujeres de nuestro estudio la existencia de un método que les permitiría tomar el control frente a la negativa del varón a cuidarse. Aunque ninguna de las mujeres lo había utilizado y que varias, en particular las adultas, expresaron dudas respecto de la posibilidad de colocárselo correctamente, las mujeres espontáneamente refirieron conocer la existencia de este método y manifestaron su aprobación a esta alternativa. (Los varones entrevistados, en cambio, manifestaron desconocimiento y actitudes reticentes o francamente negativas frente al preservativo femenino).

En síntesis, ser sensible a la manera en que la población percibe y conceptualiza las ETS/el VIH-SIDA y a las complejas negociaciones (con uno mismo y con el otro) que el "sexo más seguro" implica son requisitos fundamentales para el diseño de actividades de prevención que interpelen más eficazmente a la población.

\section{Referencias}

CAMPBELL, C., 1995. Male gender roles and sexuality: implications for women's AIDS risk and prevention. Social Science and Medicine, 41:197-210.

CAROVANO, K., 1995. VIH/SIDA y los desafíos que enfrentan los hombres. Implicaciones para un cambio de conducta. Desidamos, 3:17-20.

DÉSORMEAUX, J.; ZALDUONDO, B.; FLAMBERT, M. C.; BERNARD, J. M.; CELENTANO, D. A.; MAYARD, G.; NATHANSON, C. A.; HOLT, E.; BOULOS, R. \& HALSEY, N. A., 1992. The importance of local concepts of contagion and sexual conduct for AIDS education: A case from urban Haiti. Centres pour le Développement et la Santé (CDS), Port-auPrince, Haiti. Baltimore: Johns Hopkins University School of Hygiene and Public Health. (mimeo)

DIXON-MUELLER, R., The sexuality connection in reproductive health. Studies in Family Planning, 245:269-282.

FOSTER, G. \& ANDERSON, B., 1978. Medical Anthropology. New York: Alfred Knopf.
GUPTA, G. \& WEISS, E., 1993. Women and AIDS: Developing a New Health Strategy. New York: ICRW Policy Series.

HELITZER-ALLEN, D. \& ALLEN, H. 1994. The Manual for Targeted Intervention Research on Sexually Transmitted Illnesses with Community Members. Baltimore: Allen and Associates.

HELITZER-ALLEN, D.; MAKHAMBERA, M. \& WANGEL, A., 1994. Obtaining Sensitive Information: The Need for More than Focus Groups. Reproductive Health Matters, 3:75-82.

PAIVA, V., 1993. Sexuality, condom use and gender norms among Brazilian teenagers. Reproductive Health Matters, 2:98-109.

ZELLER, R., 1990. Qualitative approaches to the study of human sexuality. In: Human Sexuality: Research Perspectives in a World Facing AIDS. (A. Chouinard \& J. Albert, eds.), pp. 47-68. International Development Research Centre. 